\title{
Politique
}

Politique

\section{Solde budgétaire et cycle électoral au Canada et aux États-Unis}

\section{Richard Nadeau}

Numéro 18, automne 1990

URI : https://id.erudit.org/iderudit/040669ar

DOI : https://doi.org/10.7202/040669ar

Aller au sommaire du numéro

Éditeur(s)

Société québécoise de science politique

ISSN

0711-608X (imprimé)

1918-6584 (numérique)

Découvrir la revue

Citer cet article

Nadeau, R. (1990). Solde budgétaire et cycle électoral au Canada et aux États-Unis. Politique, (18), 63-81. https://doi.org/10.7202/040669ar

\section{Résumé de l'article}

La thèse de la manipulation des revenus et des dépenses par les gouvernements à des fins électorales a rencontré récemment de nombreux contradicteurs. La présente étude propose une réévaluation des conclusions d'une recherche représentative de ce courant, celle de David Lowery portant sur les soldes budgétaires aux États-Unis. Elle prend le contrepied de la conclusion principale de cet auteur en démontrant l'existence de ces manipulations au Canada et aux États-Unis au cours des dernières décennies. 


\title{
Solde budgétaire et cycle électoral au Canada et aux États-Unis
}

\author{
Richard Nadeau \\ Université de Montréal
}

La thèse de la manipulation des revenus et des dépenses par les gouvernements à des fins électorales, thèse classique du journalisme politique, a trouvé audience auprès des spécialistes des sciences sociales depuis la publication des travaux théoriques de Nordhaus (1975) et des travaux empiriques de Tufte (1978). On l'invoque maintenant régulièrement pour expliquer la croissance des dépenses gouvernementales (Cameron, 1978) ou les ratés de la gestion macroéconomique des deux dernières décennies (Willet, 1989).

Bien qu'elle soit répandue, cette thèse, aussi appelée thèse du cycle électoral, ne fait cependant pas l'unanimité. Un courant de recherches met en doute l'importance, sinon l'existence, de manipulations budgétaires (Paldam, 1981, 1989). Une des études les plus représentatives de ce courant est celle de David Lowery (1985) sur les soldes budgétaires fédéraux aux États-Unis entre 1954 et 1983 . Au terme de son analyse, Lowery conclut: «The results provide little support for the electoral motivation... interprétation of the political business cycle» (p. 429). 
La présente étude entend réexaminer cette conclusion en élargissant la période étudiée par Lowery et en comparant les cas canadien et américain. Elle vise à vérifier si l'évolution des soldes budgétaires des gouvernements fédéraux de ces deux pays confirme ou infirme la thèse du cycle électoral. Elle s'inscrit dans le contexte d'un débat qui, de l'avis de plusieurs, est loin d'être clos.

\section{Le débat autour de la thèse du cycle électoral}

Les travaux empiriques de Tufte (1978), de même que ceux de Frey et Schneider (1978a, b), ont beaucoup contribué en un premier temps à diffuser la thèse du cycle électoral dont ils semblaient démontrer avec assez de netteté qu'elle s'appliquait à plusieurs pays et à plusieurs définitions de la variable dépendante observée: dépenses, revenus, solde budgétaire, dépenses globales ou dépenses sectorielles. Mais très vite, la thèse du cycle électoral a rencontré des contradicteurs. Brown et Stein (1982), Paldam (1981) et Golden et Poterba (1980) ont mis en doute la méthodologie et les résultats des premières études. Ces critiques ont favorisé alors l'expression d'un scepticisme certain envers la thèse des manipulations budgétaires.

Un dernier virage s'est amorcé au milieu des années 80 , cette fois dans le sens d'un regain de faveur mitigé à l'égard de la thèse du cycle électoral. Une première série de travaux de recherche ont essayé de démontrer l'existence de manipulations budgétaires à visées électorales aux États-Unis et en Israël (Borjas, 1984; Kiewett et McCublins, 1985; Mintz, 1988). Se sont ensuite ajoutés d'autres travaux qui nuancent ou contestent les études importantes de Lowery (1985) et Beck (1987), lesquelles avaient conclu à l'absence de telles manipulations pour ce qui est des politiques fiscale et monétaire américaines. Par exemple, Hibbs (1987) laisse entendre que les manipulations sont réelles, mais non systématiques. Grier (1989) prend nettement le contrepied de la conclusion de Beck en affirmant que: «Presidential elections significantly influence money growth even when allowing for the effects of interest rates, deficit, and GNP» (p. 389). 
Au-delà de ce débat, on peut dire que les propos des tenants de la thèse du cycle électoral sont passés de la certitude à la modération en ce qui concerne l'ampleur des manipulations électorales. Leur argumentation tient maintenant compte des contraintes bureaucratiques, économiques et institutionnelles qui limitent les manoeuvres de réélection des politiciens (Alesina, 1988). Désormais on s'entend pour dire que, si les manipulations budgétaires à caractère électoral existent, elles sont limitées (1 à 3\% du total des dépenses gouvernementales). Les études canadiennes en sont arrivées elles aussi à la même conclusion tant au niveau des provinces (Simeon et Miller, 1980; Blais et Nadeau, 1990) que du gouvernement fédéral (Maslove, 1985; Cameron, 1986).

Ce bref état de la question semble donner un certain poids à l'hypothèse de l'existence de manipulations budgétaires réelles, mais limitées. Nous allons le vérifier à l'aide des soldes budgétaires des administrations fédérales canadienne et américaine en nous servant du modèle de David Lowery.

\section{Le modèle de Lowery}

Les travaux de David Lowery appartiennent à une série d'études qui visent à vérifier l'existence et à préciser le sens de ce qu'on a appelé un «political business cycle» (Nordhaus, 1975), expression qui signifie simplement qu'en démocratie la conjoncture économique est en partie le reflet de considérations électorales. L'existence d'un pareil cycle suppose que les gouvernements orchestrent la politique de gestion macroéconomique de façon à maximiser leurs chances de réélection. L'aménagement optimal du calendrier de la politique de stabilisation économique, dans le contexte d'un mandat gouvernemental, serait le suivant: durant la première partie du mandat, l'objectif privilégié serait la lutte à l'inflation, et l'orientation de la politique économique serait restrictive; durant la seconde partie du mandat, la priorité serait accordée au chômage, et l'orientation de la politique économique deviendrait expansionniste. On connaîtrait donc d'abord la coexistence d'un taux d'inflation faible et d'un taux de chômage élevé; la situation 
se renverserait ensuite: taux de chômage bas et taux d'inflation élevé. Ce renversement correspond précisément à la notion de «political business cycle».

Le scénario que nous venons de décrire suppose: que les gouvernements se livrent effectivement à des manipulations budgétaires à des fins électorales; que ces manipulations modifient de façon significative les données de la conjoncture économique et que les électeurs sont influencés dans leur choix électoral par le rendement de l'économie au cours d'une période allant de six mois à un an avant la tenue d'un scrutin 1 .

L'articulation des composantes ci-dessus a donné un modèle dit politico-économique. Toutefois, la plupart des études qui se rapportent à l'interaction élus/électeurs opérée par le biais de la conjoncture économique ne portent que sur l'un ou l'autre aspect de ce modèle. On peut ainsi distinguer trois types d'études. Un premier type examine la fonction de réaction des gouvernements et pose la question de l'existence et de l'ampleur des manipulations budgétaires électoralistes. C'est à cette catégorie de travaux que se rattachent l'étude de Lowery et le présent texte. Un deuxième type de travaux renvoie à un long débat entre économistes sur la capacité des gouvernements de modifier de façon significative les données de la conjoncture économique de court terme. Le troisième type essaie de mesurer l'impact des conditions économiques sur les choix électoraux (Hibbs, 1982).

Pour sa part, Lowery ne s'est donc intéressé qu'à l'existence et à l'ampleur des manipulations budgétaires à visées électorales. Le choix de la politique fiscale comme objet d'études, et du solde budgétaire en particulier, se comprend aisément. D'une part, les

1. De façon plus formelle, les trois conditions précédentes supposent l'existence: (1) d'une fonction de réaction des gouvernements reflétant leur volonté de modifier la conjoncture économique dans une perspective électoraliste, (2) d'une relation d'arbitrage de court terme entre le taux de chômage et le taux d'inflation (relation de Phillips), (3) d'une fonction d'évaluation de la performance gouvernementale chez les électeurs, caractérisée par l'influence réelle des considérations économiques de court terme sur les choix électoraux. 
économistes admettent généralement que la politique fiscale est la mieux adaptée (et la plus utilisée) pour gérer la conjoncture économique domestique d'un pays - dont dépendent surtout les choix électoraux - et pour composer avec le problème du chômage (Samuelson, 1983). D'autre part, le solde budgétaire d'un gouvernement, parce qu'il est le résultat de décisions prises tant au chapitre des revenus qu'à celui des dépenses, constitue la caisse de résonance par excellence des manipulations budgétaires.

Mais comme les considérations électorales ne sont qu'un des déterminants du solde budgétaire d'un gouvernement, l'étude de leur impact doit se faire dans le cadre d'un modèle d'explication global des soldes budgétaires. Selon Lowery, et nous souscrivons à son argumentation, à court terme, les facteurs décisifs expliquant l'évolution de cette variable relèvent de la politique de stabilisation économique. Celle-ci comprend deux composantes: l'une liée à l'action des stabilisateurs automatiques de la politique macroéconomique et l'autre relevant plutôt de l'orientation discrétionnaire de cette politique (Blinder et Solow, 1973).

Par conséquent, le modèle d'analyse devra permettre de départager les effets respectifs des manipulations électorales et ceux de la politique macroéonomique sur les soldes budgétaires gouvernementaux. Le modèle proposé par Lowery, et c'est là son intérêt majeur, permet de répondre à ces exigences avec économie. L'équation (1) en résume les aspects essentiels 2 :

(1) Solde

$$
\begin{aligned}
\text { budgétaire }_{\mathrm{t}} & =\alpha_{0}+\mathrm{B}_{1} \mathrm{TCH}_{\mathrm{t}-1}+\mathrm{B}_{2} \varnothing \mathrm{TCH}+\mathrm{B}_{3} \mathrm{MD}_{\mathrm{t} 1}+\mathrm{B}_{4} \mathrm{MD}_{\mathrm{t} 2} \\
& +\mathrm{B} 5 \mathrm{MD}_{\mathrm{t} 3} \\
& \text { où }
\end{aligned}
$$

- $\mathrm{TCH}_{\mathrm{t}-1}=$ taux de chômage au temps $\mathrm{t}-1$; cette variable reflète la composante discrétionnaire de la politique de stabilisation économique (on suppose ici que le gouvernement

2. Des adaptations mineures, essentiellement au niveau des symboles et du choix de la catégorie manquante des variables démarquant les années des mandats gouvernementaux, sont apportées ici. Elles n'affectent en rien la comparabilité de l'équation (1) de notre texte et de l'équation (3a) du tableau 1 du texte de Lowery (p. 446). 
visera au temps $\mathrm{t}$ l'atteinte d'un taux donné de chômage $\mathrm{TCH} * \mathrm{t}$; l'écart entre $\mathrm{TCH} \mathrm{t}-1$ et $\mathrm{TCH}{ }^{*} \mathrm{t}$ déterminera donc l'orientation de la politique fiscale discrétionnaire au temps t).

$\nabla \quad \varnothing \mathrm{TCH}=$ variation des taux de chômage entre les temps t et $\mathrm{t}-1$; cette variable reflète la composante automatique de la politique de stabilisation économique.

- $\quad \mathrm{MD}_{\mathrm{t} 1, \mathrm{t} 2, \mathrm{t3}}=$ variables dichotomiques démarquant les années post-électorales, intermédiaires et préélectorales d'un mandat gouvernemental.

On notera tout d'abord que le modèle de Lowery inclut la variable chômage en raison des effets direct et indirect des variations du taux de chômage sur les soldes budgétaires gouvernementaux. Par effet direct, on entend, par exemple, l'augmentation ou la diminution des paiements de transfert liés au sous-emploi, conséquence qui renvoie à la dimension automatique de la politique de stabilisation économique. Par effet indirect, on entend les décisions que prennent les gouvernements en réaction aux fluctuations économiques, conséquence qui fait appel à la composante discrétionnaire de la gestion macroéconomique.

Il faut ensuite souligner que Lowery a ajouté à son modèle général un certain nombre de variables permettant de prendre en compte d'éventuelles différences dans les résultats obtenus, différences attribuables aux administrations ou à la couleur politique de l'équipe présidentielle. Cependant, l'inclusion de ces variable n'a affecté que de façon marginale les résultats de son étude.

L'économie du modèle de Lowery, sa valeur explicative, le caractère systématique et la densité de ses travaux ${ }^{3}$ de même que la convergence de ses résultats avec ceux des importantes études de

3. Lowery a examiné un très grand nombre d'hypothèses portant sur l'existence d'une interaction entre les aspects partisans et électoraux des manipulations budgétaires. Ces tests, non concluants pour la plupart dans le cas américain, n'ont pu être repris dans le cas canadien étant donné le quasi-monopole que détenait le Parti libéral à titre de parti gouvernemental au cours de la période étudiée. 
Golden et Poterba (1980) et de Hibbs (1987) 4 nous ont fait opter pour ce modèle afin de comparer les cas canadien et américain.

Mais avant d'aborder la question sous l'angle comparatif, il convient d'examiner les résultats qu'obtient Lowery. Ils peuvent se résumer ainsi: 1) les deux coefficients associés à la politique de stabilisation économique sont de signe attendu, c'est-à-dire négatif, et ils sont statistiquement significatifs et d'amplitude semblable; 2) les coefficients associés aux manipulations budgétaires sont de signes attendus, d'amplitude semblable, mais ne sont pas statistiquement significatifs. C'est à la lumière de cette dernière donnée que Lowery conclut à la quasi-absence de l'impact des considérations électorales sur les soldes budgétaires des administrations fédérales américaines au cours de la période 1954-1983.

Cette conclusion nous semble excessive. Tout d'abord, elle ne tient pas compte du nombre de cas à l'étude, une variable liée positivement au seuil de signification statistique des coefficients obtenus (Lewis-Beck, 1980). Ensuite, elle ne met pas suffisamment en perspective les contraintes bureaucratiques, économiques et institutionnelles qui peuvent rétrécir la marge de manoeuvre des gouvernements et qui font en sorte que les manipulations budgétaires dont font état les études récentes ne peuvent que constituer un effet à la marge dans l'ensemble des facteurs qui affectent les soldes budgétaires gouvernementaux. Finalement, compte tenu des observations précédentes, elle ne mentionne pas que le résultat

4. Les résultats de Golden et Poterba (voir en particulier le Tableau 3 de leur étude) et ceux de Hibbs (voir les conclusions qu'il tire de ceux-ci à la page 262 de son ouvrage) comme ceux de Lowery sont conformes à la thèse du cycle électoral sans atteindre toutefois les seuils de signification statistique usuels. Golden et Poterba, de même que Lowery, voient dans ceux-ci l'infirmation de la thèse des manipulations budgétaires alors que Hibbs s'attache à démontrer que ces manipulations ont été le fait de certains présidents. C'est la convergence des résultats obtenus, malgré l'emploi de modèles différents, qui doit retenir l'attention. Nous expliquons plus loin pourquoi ces résultats nous semblent apporter de l'eau au moulin à la proposition voulant que les manipulations budgétaires soient réelles, mais circonscrites et limitées. 
obtenu par Lowery est tout à fait plausible, à savoir que des coefficients de signe attendu peuvent ne pas être significatifs sur le plan statistique. C'est pour ces raisons que nous avons jugé bon de reprendre l'analyse de Lowery en lui apportant les trois modifications suivantes:

- Le cas américain est comparé au cas canadien pendant deux périodes quasi identiques (1948-1984 dans le premier cas et 1949-1984 dans le second);

- $\quad$ Pour compenser le petit nombre d'observations, nous avons élargi la période d'étude en incluant les années du second mandat Truman; cela a permis de serrer la chronologie en respectant la logique du découpage de la période en mandats gouvernementaux (du deuxième mandat Truman au premier mandat Reagan pour les États-Unis, et du premier mandat Saint-Laurent au dernier mandat Trudeau pour le Canada);

- $\quad$ Afin de rendre la lecture et l'interprétation des résultats plus faciles et plus suggestives, nous avons: a) établi nos résultats sur la base de l'année civile; b) retenu l'année électorale comme catégorie manquante dans la construction des variables démarquant les différentes années des mandats gouvernementaux; c) défini la variable «solde budgétaire» en dollars constants $(1981=100)$, mais sans autre transformation, ce qui permet, sans grand inconvénient économétrique, d'interpréter de façon simple et claire les coefficients obtenus.

\section{Modèle et résultats}

Le modèle utilisé reposera donc sur les fondements théoriques de Lowery. D'une part, l'inclusion des deux variables concernant le chômage $\left(\mathrm{TCH}_{\mathrm{t}-1}\right.$ et $\left.\varnothing \mathrm{TCH}\right)$ renverra aux conséquences budgétaires liées à la pratique d'une politique de stabilisation économique. D'autre part, la présence de variables dichotomiques associées aux diverses années des mandats gouvernementaux (l'année électorale $\mathrm{MD}_{\mathrm{t} 0}$ étant définie comme la catégorie manquante) 
renverra à la thèse du cycle électoral. En bref, l'équation de régression type qui sera étudiée prendra la forme suivante:

(2) Solde

budgétaire ${ }_{\mathrm{t}}=\alpha_{0}+\mathrm{B}_{1} \mathrm{TCH}_{\mathrm{t}-1}+\mathrm{B}_{2} \varnothing \mathrm{TCH}+\mathrm{B}_{3} \mathrm{MD}_{\mathrm{t} 1}+\mathrm{B}_{4} \mathrm{MD}_{\mathrm{t} 2}$ (Can., É.-U.) + B5 MD $\mathrm{MD}_{\mathrm{t}}$

Pour compléter le modèle général de Lowery, nous avons inclus des variables dichotomiques qui tiennent compte de l'évolution budgétaire particulière des deux pays à l'étude. Dans le cas canadien, il semble exister un consensus à l'effet que la période postérieure à 1975 en a été une d'explosion du déficit budgétaire (Purvis et Smith, 1986). Une variable dichotomique isolant cette période a donc été incluse dans l'équation de régression. Dans le cas américain, en l'absence d'un consensus aussi clair, une approche plus inductive a été adoptée. Elle nous a amené à isoler les années 1982-1984 du reste de la période ${ }^{5}$. Les équations 3 et 4 ont été finalement retenues.

5. Ces années ont été marquées par un décrochage sans précédent dans l'histoire budgétaire récente des États-Unis entre les revenus et les dépenses de l'administration fédérale. Les données cidessous montrent l'ampleur de ce décrochage (il s'agit de données se rapportant à l'administration fédérale américaine; ces données sont exprimées en milliards de dollars, 1982-1984 = 100).

$\begin{array}{cccc} & \text { Dépenses } & \text { Revenus } & \text { Solde budgétaire } \\ 1980 & 615.1 & 553.8 & -61.3 \\ 1981 & 703.3 & 639.5 & -63.8 \\ 1982 & 781.2 & 635.3 & -145.9 \\ 1983 & 835.9 & 659.9 & -176.0 \\ 1984 & 893.6 & 726.0 & -169.6\end{array}$

Source: US Department of Commerce, Business Conditions Digest, mars 1989. 
(3) Solde

budgétaire $=\alpha_{0}+\mathrm{B}_{1} \mathrm{TCH}_{\mathrm{t}-1}+\mathrm{B}_{2} \varnothing \mathrm{TCH}+\mathrm{B}_{3} \mathrm{MD}_{\mathrm{t} 1}+\mathrm{B}_{4} \mathrm{MD}_{\mathrm{t} 2}$

(Canada) $+\mathrm{B} 5 \mathrm{MD}_{\mathrm{t} 3}+\mathrm{B}_{6} \mathrm{PER}_{7584}$

(4) Solde

budgétaire $=\alpha_{0}+\mathrm{B}_{1} \mathrm{TCH}_{\mathrm{t}-1}+\mathrm{B}_{2} \varnothing \mathrm{TCH}+\mathrm{B}_{3} \mathrm{MD}_{\mathrm{t} 1}$

(États-Unis) $+\mathrm{B}_{4} \mathrm{MD}_{\mathrm{t} 2}+\mathrm{B}_{5} \mathrm{MD}_{\mathrm{t} 3}+\mathrm{B}_{6} \mathrm{PER}_{8284}$

Les résultats de notre étude sont présentés au Tableau 1. Ils ont été obtenus par l'utilisation de la méthode de régression dite des moindres carrés ordinaires avec correction de l'autocorrélation des erreurs résiduelles (Johnston, 1984).

Les résultats ${ }^{6}$ montrent l'effet du chômage sur le solde budgétaire dans les deux pays et signalent l'évolution particulière de celui-ci au cours des deux sous-périodes mentionnées précédemment. À propos des variables électorales qui nous intéressent plus particulièrement, deux observations découlent de l'examen des équations 3 et 4.

Dans les deux pays, le coefficient associé à l'année préélectorale (t3) s'avère faible (quoique de signe attendu), ce qui signale la similitude entre celle-ci et l'année électorale (t0); l'hypothèse

6. Les coefficients du Tableau 1 peuvent être interprétés de la façon suivante. Les chiffres correspondant aux variables $\mathrm{TCH}_{\mathrm{t}-1}$ et $\Delta \mathrm{TCH}$ représentent l'effet qu'entraînerait sur le solde budgétaire des gouvernements canadien et américain une hausse de $1 \%$ du taux de chômage au cours d'une année donnée. Les coefficients associés aux variables renvoyant à des sous-périodes particulières représentent la variation des soldes budgétaires, attribuable à des variables non incluses dans le modèle (réforme fiscale, chute de la productivité, ralentissement de la croissance économique, etc.), et dont l'effet se serait manifesté durant ces sous-périodes. Finalement, les coefficients des variables $\mathrm{MD}_{\mathrm{t} 1},{ }_{\mathrm{t} 2}$ et ${ }_{\mathrm{t} 3}$ signalent l'effet des manipulations budgétaires. Dans le cas canadien, on constate par exemple qu'un déficit du gouvernement fédéral serait plus élevé au cours des années préélectorales et électorales que durant les années post électorales et intermédiaires (l'écart observé étant de plus de 1 milliard de dollars, 1981=100; voir l'équation 3a). 
voulant que l'année électorale se démarque de toutes les autres n'est donc pas confirmée pour la période à l'étude.

Tableau 1: Résultats d'une analyse de régression sur les déterminants du solde budgétaire du gouvernement fédéral au Canada (1949-1984) et aux États-Unis (1948-1984).

CANADA

ÉTATS-UNIS

\begin{tabular}{|c|c|c|c|c|}
\hline Variables & Equation 3 & Équation 3a & Équation 4 & Equation 4a \\
\hline Constante & $\begin{array}{c}7105,0 \\
(5,89)^{2}\end{array}$ & $\begin{array}{c}7262,9 \\
(5,88)^{2}\end{array}$ & $\begin{array}{c}35117,1 \\
(1,44)^{\mathrm{c}}\end{array}$ & $\begin{array}{c}36566,7 \\
(1,54)^{c}\end{array}$ \\
\hline \multirow[t]{2}{*}{$\mathrm{TCCH}_{\mathrm{t}-1}$} & $-1476,7$ & $-1465,1$ & $-12350,9$ & $-12145,7$ \\
\hline & $(-6,58)^{2}$ & $(-6,87)^{2}$ & $(-2,95)$ & $(-2,99)^{2}$ \\
\hline THC & $\begin{array}{c}-1300,2 \\
(-4,76)^{2}\end{array}$ & $\begin{array}{c}-1242,6 \\
(-4,80)^{2}\end{array}$ & $\begin{array}{c}-15784,1 \\
(-4,54)^{2}\end{array}$ & $\begin{array}{c}-15295,6 \\
(-4,56)^{2}\end{array}$ \\
\hline PER 7584 & $\begin{array}{c}-5375,2 \\
(-4,69)^{2}\end{array}$ & $\begin{array}{c}-5335,1 \\
(-4,77)^{2}\end{array}$ & $\begin{array}{c}-88 \quad 338,2 \\
(-3,92)^{2}\end{array}$ & $\begin{array}{c}-86701,4 \\
(-4,01)^{\mathbf{a}}\end{array}$ \\
\hline \multirow[t]{2}{*}{$\mathrm{MD}_{\mathrm{t}}$} & 1261,3 & - & 12645,4 & - \\
\hline & $\begin{array}{l}(1,99)^{b} \\
1362,0\end{array}$ & - & $\begin{array}{l}(1,41)^{c} \\
1885,0\end{array}$ & - \\
\hline $\mathrm{MD}_{12}$ & $\begin{array}{c}(1,79)^{\mathrm{b}} \\
(707,7\end{array}$ & - & $\begin{array}{l}(1,84)^{b} \\
5494,2\end{array}$ & - \\
\hline $\mathrm{MD}_{\mathrm{L} 3}$ & $(0,95)$ & - & $(0,62)$ & - \\
\hline $\mathrm{MD}_{\mathrm{t} 1 \mathrm{1}}$ & - & $\begin{array}{l}1074,6 \\
(2,04)^{b}\end{array}$ & - & $\begin{array}{c}12850,6 \\
(1,83)^{b}\end{array}$ \\
\hline $\mathrm{R} 2$ & 0,88 & 0,89 & 0,63 & 0,65 \\
\hline DW & 1,87 & 1,84 & 1,47 & 1,46 \\
\hline Rho & 0,29 & 0,26 & 0,44 & 0,44 \\
\hline $\mathbf{N}$ & 35 & 35 & 36 & 36 \\
\hline
\end{tabular}

1. Les chiffres entre parenthèses sont les statistiques $t$ de Student. Les seuils de signification statistique correspondants sont les suivants:

a) $99 \%$ (test unilatéral); b) $95 \%$ (test unilatéral); c) $90 \%$ (test unilatéral)

2. Les coefficients Rho ont été obtenus en appliquant la méthode de correction de l'autocorrélation des erreurs résiduelles dite de Cochrane-Orcutt 
Les coefficients associés aux années postélectorales (t1) et intermédiaires ( $t 2)$ sont tous de signe attendu et sont statistiquement significatifs, ce qui suggère un certain découpage des mandats électoraux du point de vue des manipulations budgétaires.

Ce redécoupage a pour effet de diviser en deux les mandats de quatre ans ${ }^{7}$ en démarquant les années postélectorales et intermédiaires ( $t 1$ et $t 2)$ des années préélectorales et électorales ( $\mathrm{t}$ et $t 0)$.

Les résultats ci-dessus nous ont amené à examiner des versions remaniées des équations 3 et 4 . Ces versions sont les suivantes:

7. Les mandats électoraux américains ayant une durée fixe de quatre ans, aucun problème ne se pose dans ce pays quant à la définition des différentes années $(\mathrm{t} 0, \mathrm{t} 1, \mathrm{t} 2, \mathrm{t})$ correspondant au cycle électoral. Le cas canadien est plus complexe. Six des douze mandats au cours de la période ont eu une durée approximative de quatre ans (1949-1953, 1953-1957, 1958-1962, 1968-1972, 1974-1979, 19801984), ces mandats recoupant 30 des 36 années à l'étude, soit près de $85 \%$ de la période étudiée. Les très courts mandats de John Diefenbaker (1957-1958, 1962-1963) et celui non moins bref de Joe Clark (19791980) ne posent pas non plus de problèmes de définition puisqu'ils ont chevauché des années électorales. Restent les cas plus problématiques des mandats Pearson $(1963-1965 ; 1965-1968)$ et du deuxième mandat Trudeau (1972-1974). Une lecture de la conjoncture politique canadienne (Saywell, 1963-1969) nous fait conclure à une situation de quasi-majorité dans le cas des mandats Pearson, ce qui nous amène à considérer 1964, 1966, 1967 comme des années postélectorales et intermédiaires plutôt que comme des années préélectorales. Le cas de 1973 est beaucoup plus ambigu. Nous l'avons finalement définie comme une année postélectorale bien qu'ici ce choix ne modifie que marginalement les résultats obtenus. Reste le cas de l'année 1978, cinquième année d'un mandat de cinq ans. Ici encore des données circonstancielles laissent supposer que 1978 avait probablement été prévue d'abord comme devant être une année électorale. Des sondages défavorables (Peniman, 1981) ont repoussé cette échéance à 1979. Dans ce cas très particulier, 1977 et 1978 ont été définies comme années préélectorales. 
(3a) Solde

$$
\begin{aligned}
\text { budgétaire }= & \alpha_{0}+\mathrm{B}_{1} \mathrm{TCH}_{\mathrm{t}-1}+\mathrm{B}_{2} \varnothing \mathrm{TCH}+\mathrm{B}_{3} \mathrm{PER}_{7584} \\
\text { (Canada) } & +\mathrm{B} 4 \mathrm{MD}_{\mathrm{t} 1, \mathrm{t} 2}
\end{aligned}
$$

(4a) Solde

$$
\begin{aligned}
& \text { budgétaire }=\alpha_{0}+\mathrm{B}_{1} \mathrm{TCH}_{\mathrm{t}-1}+\mathrm{B}_{2} \varnothing \mathrm{TCH}+\mathrm{B}_{3} \mathrm{PER}_{8284} \\
& \text { (États-Unis) }+\mathrm{B} 4 \mathrm{MD}_{\mathrm{t} 1, \mathrm{t} 2}
\end{aligned}
$$

Les résultats des équations 3a et 4a confirment de façon un peu plus nette l'existence de manipulations budgétaires à des fins électorales tant au Canada qu'aux États-Unis.

Il convient de s'interroger sur l'ampleur de ces manipulations budgétaires. À l'aide des coefficients des équations $3 \mathrm{a}$ et $4 \mathrm{a}$, on peut établir que les déficits des gouvemements canadien et américain auraient augmenté de plus de 1 milliard et demi de dollars au Canada et de quelque 16 milliards de dollars aux États-Unis (en dollars de 1989) durant les années préélectorales et électorales par rapport aux années intermédiaires et postélectorales. En moyenne, au cours de la période à l'étude, ces manipulations auraient représenté un peu moins de 3\% (2,8\% dans chaque cas) des dépenses des administrations fédérales canadienne et américaine.

En concluant, il faut également s'interroger sur l'importance du phénomène observé. On doit se rappeler que les pourcentages évoqués ci-dessus représentent l'effet total des manipulations budgétaires. Cet effet englobe des manipulations qui ont pu porter à la fois sur les revenus et les dépenses. Cela explique que les résultats qui appuient le mieux la thèse du cycle électoral aient été obtenus en examinant les soldes budgétaires plutôt que les revenus ou les dépenses séparément, l'étude directe de ces variables ayant souvent débouché sur des conclusions plus mitigées. Les manipulations budgétaires sont donc difficilement perceptibles dans ces circonstances, ce qui précise le sens de l'expression «manipulations budgétaires limitées» utilisée dans la présente étude.

Enfin, si nos résultats diffèrent de ceux de Lowery, c'est en partie parce que la période à l'étude est différente et parce que les variables démarquant des sous-périodes particulières ne sont pas les mêmes dans les deux cas. Ce simple fait montre à lui seul que les 
manipulations budgétaires dont il a été question dans ce texte n'ont pas le caractère systématique et l'ampleur qu'on a voulu leur prêter.

Le débat sur la thèse du cycle électoral est loin d'être clos, ni dans son aspect théorique, ni dans son aspect empirique. La polémique autour du profil de l'électeur, dont dépend la pertinence des manipulations budgétaires, a évolué depuis quinze ans. En effet, si la notion de l'électeur naif et myope de Nordhaus a été délaissée, on ne souscrit plus par ailleurs avec le même enthousiasme à celle de l'électeur rationnel disposant d'une information complète sur les grandes données de son environnement macroéconomique. Les travaux les plus récents sur la question adoptent plutôt un point de vue intermédiaire: l'électeur serait rationnel mais imparfaitement informé, caractérisation qui inciterait les gouvernements aux manipulations budgétaires (Rogoff, 1990).

Le volet empirique du débat, qui nous intéressait plus particulièrement, a connu lui aussi une grande évolution. On a d'abord parlé de manipulations relativement importantes et systématiques. Le point de vue fut ensuite durement contesté. Là encore, les travaux les plus récents ont adopté une perspective nuancée en essayant de démontrer que les manipulations budgétaires des gouvernements sont bien réelles, mais qu'elles sont modestes compte tenu des contraintes administratives, économiques et institutionnelles qui limitent ce genre de pratiques.

La présente étude s'appuyait sur ces acquis théoriques et empiriques. Son hypothèse centrale était l'existence de manipulations budgétaires réelles mais limitées. Cette proposition a été mise à l'épreuve par l'examen de l'évolution des soldes budgétaires canadien et américain au cours des dernières décennies. Les résultats obtenus ont confirmé dans l'ensemble cette hypothèse. Ils constituent donc un élément de preuve supplémentaire à l'appui de la thèse voulant que les pratiques fiscales électoralistes soient réelles, mais circonscrites et limitées. 


\section{Bibliographie}

ALESINA, Alberto , «Macroeconomics and Politics». dans Stanley FISCHER, NBER Macroeconomics Annual, Cambridge, MIT Press, 1988, pp. 13-65.

BECK, Nathaniel, «Elections and the Fed: Is There a Political Monetary Cycle?» American Journal of Political Science, vol. 31, 1987, pp. 194-216.

BLAIS, André, et NADEAU, R., The Electoral Cycle Thesis, Manuscrit non publié, Département de science politique, Université de Montréal, 1990.

BLINDER, Alan S., et SOLOW, R., «Analytical Foundations of Fiscal Policy», dans The Economics of Public Finance, Washington, D.C., Brookins Institution, 1973, pp. 3-115.

BORJAS, George J., «Electoral Cycles and the Earnings of Federal Bureaucrats», Economic Inquiry, vol. 22, 1984, pp. 447-460.

BROWN, Thad A. et STEIN, Arthur A., «The Political Economy of National Elections», Comparative Politics, vol. 14, 1982, pp. 479-499.

CAMERON, David R., «The Expansion of the Public Economy: A Comparative Analysis», American Political Science Review, vol. 72, 1978, pp. 1243-1261.

CAMERON, David R., «The Growth of Government Spending: The Canadian Experience in Comparative Perspective», dans Keith Banting State and Society: Canada in Comparative Perspective, Toronto, University of Toronto Press, 1986, pp. 21-51.

FREY, Bruno S. et SCHNEIDER, F., «An Empirical Study of Politico-Economic Interaction in the United States», Review of Economics and Statistics, vol. 60, 1978a, pp. 174-184.

FREY, Bruno S. et SCHNEIDER, F., «A Politico-Economic Model of the United Kingdom», Economic Journal, vol. 88,1978 b, pp. 243-254. 
GOLDEN, D.G. et POTERBA, J. M., «The Price of Popularity: The Political Business Cycle Reexamined», American Journal of Political Science, vol. 24, 1980, pp 696-714.

GRIER, Kevin B., "On the Existence of a Political Monetary Cycle», American Journal of Political Science, vol. 33, 1989, pp 376-390.

HIBBS, Douglas A., «On the Demand for Economic Outcomes: Macroeconomic Performance and Mass Political Support in the United States, Great Britain and Germany», Journal of Politics, vol. 44, 1982, pp 426-462.

HIBBS, Douglas A., The American Political Economy: Macroeconomics and Electoral Politics in the United States, Cambridge, Harvard University Press, 1987.

JOHNSTON, J., Econometric Methods (3e édition), New-York, McGraw-Hill, 1984.

KIEWETT, D. Roderick et McCUBLINS, Matthew D., «Congressional Appropriations and the Electoral Connection», Journal of Politics, vol. 47, 1985, pp. 5983.

LEWIS-BECK, Michael S., Applied Regression, Beverly Hills, Sage, 1980.

LOWERY, David L., «The Keynesian and Political Determinants of Unbalanced Budgets: U.S. Fiscal Policy from Eisenhower to Reagan», American Journal of Political Science, vol. 29, 1985, pp. 429-460.

MASLOVE, Allan M., PRINCE, Michael J., et DOERN, G. Bruce, Federal and Provincial Budgeting, Toronto, University of Toronto Press, 1985.

MINTZ, Alex, «Electoral Cycles and Defense Spending: A Comparison of Israel and the United States», Comparative Political Studies, vol. 21, 1988, pp. 368-381.

NORDHAUS, William, «The Political Business Cycle», Review of Economic Studies, vol. 42, 1975, pp. 169-190.

PALDAM, Martin, "An Essay on the Rationality of Economic Policy: The Test Case of an Electoral Cycle», Public Choice, vol. 37, 1981, pp. 287-307. 
PALDAM, Martin, Does Politics Matter After All? A Comparative Test on Data for 17 Countries, Note de recherche, Département d'économie, Université d'Aarhus, 1989.

PENIMAN, Howard R., Canada at Polls, 1979 and 1980: A Study of the General Elections, Washington, American Entreprise Institute, 1981.

PURVIS, D. et SMITH, Constance, «La politique budgétaire au Canada de 1963 à 1984», dans J.H. Sargent, Les politiques budgétaires et monétaires, Commission royale sur l'union économique et les perspectives de développement au Canada, Ottawa, 1986, pp. 1-50.

ROGOFF, Kenneth, «Equilibrium Political Budget Cycles», American Economic Review, vol. 80, 1990, pp. 21-36.

SAMUELSON, Paul A., Economics from the Hearth, San Diego, Calif., Harcourt, Brace, Jocanovich, 1983.

SAYWELL, John, Canadian Annual Review, Toronto, University of Toronto Press, 1963-1969.

SIMEON, Richard et MILLER, Robert E., «Regional Variations in Public Policy», dans David Elkins et Richard Simeon, (éd.) Small Worlds: Provinces and Parties in Canadian Political Life, Toronto, Methuen, 1980, pp. 242-284.

TUFTE, Edward R., Political Control of the Economy, Princeton, Princeton University Press, 1978.

WILlET, T., (éd.), Political Business Cycles: The Economics and Politics of Stagflation, San Francisco, Pacific Institute, 1989. 


\section{Annexe \\ Sources des données}

\section{A. Données canadiennes}

Solde budgétaire

Statistique Canada, Comptes nationaux des revenus et des dépenses, divers numéros.

Taux de chômage

Statistique Canada, Enquête sur la population active, divers numéros.

Années du cycle électoral:

Années électorales ( 10$)$ :

1949, 1953, 1957, 1958, 1962, 1963, 1965, 1968, 1972, 1974, 1979, 1980, 1984.

Années postélectorales (t1):

1950, 1954, 1959, 1963, 1966, 1969, 1973, 1975, 1981.

Années intermédiaires ( 2 ):

1951, 1955, 1960, 1967, 1970, 1976, 1982.

Années préélectorales ( $\mathrm{H} 3$ ):

1952, 1955, 1961, 1971, 1977, 1978, 1983.

B. Données américaines

Solde budgétaire:

U.S. Department of Commerce, Business Conditions Digest, divers numéros. 
Taux de chômage:

U.S. Department of Commerce, Business Conditions Digest, divers numéros 